\title{
A constraint on any topological lensing hypothesis in the spherical case: it must be a root of the identity
}

\author{
B. F. Roukema
}

\author{
Toruń Centre for Astronomy, N. Copernicus University, ul. Gagarina 11, 87-100 Toruń, Poland \\ e-mail: boud@astro.uni.torun.pl
}

Received 28 September 2004 / Accepted 9 May 2005

\begin{abstract}
Three-dimensional catalogues of objects at cosmological distances can potentially yield candidate topologically lensed pairs of sets of objects, which would be a sign of the global topology of the Universe. In the spherical case (i.e. if curvature is positive), a necessary condition, which does not exist for either null or negative curvature, can be used to falsify such hypotheses, without needing to loop through a list of individual spherical 3-manifolds. This condition is that the isometry between the two sets of objects must be a root of the identity isometry in the covering space $S^{3}$. This enables numerical falsification of topological lensing hypotheses without needing to assume any particular spherical 3-manifold. By embedding $S^{3}$ in euclidean 4-space, $\mathbb{R}^{4}$, this condition can be expressed as the requirement that $M^{n}=I$ for an integer $n$, where $M$ is the matrix representation of the hypothesised topological lensing isometry and $I$ is the identity. Moreover, this test becomes even simpler with the requirement that the two rotation angles, $\theta, \phi$, corresponding to the given isometry, satisfy $\frac{2 \pi}{\theta}, \frac{2 \pi}{\phi} \in \mathbb{Z}$. The calculation of this test involves finding the two eigenplanes of the matrix $M$. A GNU General Public Licence numerical package, called eigenplane, is made available for finding the rotation angles and eigenplanes of an arbitrary isometry $M$ of $S^{3}$.
\end{abstract}

Key words. cosmology: observations - cosmological parameters - cosmic microwave background - quasars: general

\section{Introduction}

Although interest in cosmic topology is just over a century old (Schwarzschild 1900, 1998), much interest has recently developed in trying to find clues to the topology of the Universe, in particular due to WMAP cosmic microwave background observations (Spergel et al. 2003) which have been found by many to have unusual statistical properties on the largest angular scales (e.g. Spergel et al. 2003; Tegmark et al. 2003; Chiang et al. 2003; Copi et al. 2004), even though one study across a wide range of parameter space failed to find any significant signal (Cornish et al. 2004).

Moreover, both the WMAP observations and supernovae Ia redshift-magnitude tests (e.g. Perlmutter et al. 1999) suggest that the Universe is spherical, but with a radius of curvature at least as large as the horizon radius. Indeed, the analysis of Choudhury \& Padmanabhan (2003) rejects the flat universe hypothesis in favour of a spherical model at either $97 \%$ or $90 \%$ confidence levels, depending on which data set of type Ia supernovae is analysed.

In addition, some analyses (Luminet et al. 2003; Roukema et al. 2004) suggest that the spatial comoving section of the Universe is a Poincaré dodecahedral space, which is a 3-manifold with $S^{3}$ as its covering space, i.e. curvature is positive.

This type of hypothesis implies tight constraints on the curvature parameters: the non-relativistic matter density parameter, $\Omega_{\mathrm{m}}$, the cosmological constant (or quintessence constant) $\Omega_{\Lambda}$, and their sum, $\Omega_{\text {tot }}=\Omega_{\mathrm{m}}+\Omega_{\Lambda}$.

While much interest is presently focussing on microwave background analyses, only a relatively small error in the present estimates of $\Omega_{\text {tot }}$ is required in order for topological lensing of sub-microwave background objects, such as quasars, to be detectable.

For example, in the right-hand plot of Fig. 15 of Gausmann et al. (2001), it was shown that for the Poincare dodecahedral space and $\left(\Omega_{\mathrm{m}}=0.35, \Omega_{\Lambda}=0.75\right)$, i.e. for $\Omega_{\text {tot }}=1.1$, a spike in the pair separation histogram (PSH) would be expected for a catalogue containing objects in the $1<z<3$ redshift range the range of most interest for large quasar catalogues.

Is $\Omega_{\text {tot }}=1.1$ reasonable? While observations do seem to be converging closer to unity than this, there are still many uncertainties present.

For example, Myers et al. (2004) found evidence for an extended Sunyaev-Zel'dovich Effect in WMAP data out to about 1 degree from centres of $z<0.2$ clusters of galaxies, and they note that the contribution from clusters at $z>0.2$ could significantly contaminate the $l=220$ first acoustic peak, so that the uncertainties stated in estimating $\Omega_{\mathrm{tot}} \sim 1$, e.g. $\Delta \Omega_{\mathrm{tot}}=0.02$ (Spergel et al. 2003), may be considerably underestimated due to sources of systematic error such as the Sunyaev Zel'dovich Effect.

Hence, three-dimensional methods of detecting cosmic topology still remain of interest, even though consistency with 
WMAP analyses would be required for any serious 3-manifold candidates.

Given the many systematic effects, both due to observation and to physical aging of objects, in catalogues of extragalactic objects, it is quite possible that a real signal could be present, but that the statistical properties of the catalogue would be insufficient to establish statistical significance from general statistical properties of the catalogue.

Any additional tests which could either falsify or support a given topological lensing hypothesis would, therefore, be useful.

In this paper, a simple property of topological lensing, which is only valid for the spherical case, is presented as such a test.

This is the necessary condition that the mapping or isometry between the two sets of objects, of which one set is the topological image of the other, must be a whole number root of the identity in the covering space, $S^{3}$. This is because $S^{3}$ is finite, while $\mathbb{R}^{3}$ and $H^{3}$ are not.

The covering space, $S^{3}, \mathbb{R}^{3}$ or $H^{3}$, (which can be physically thought of as the apparent space) relates to the 3-manifold, $M$, (which can be physically thought of as the fundamental domain with glued faces), by $M=S^{3} / \Gamma, \mathbb{R}^{3} / \Gamma$ or $H^{3} / \Gamma$ respectively, where $\Gamma$ is a group of isometries, which can be more generally called a holonomy group ${ }^{1}$.

The reason why the condition that the mapping must be a whole number root of the identity is necessary in $S^{3}$ but not in flat or negatively curved space can equivalently be understood as the fact that the holonomy group $\Gamma$ is finite for $S^{3}$, but infinite in the other two cases.

Testing this property enables numerical falsification of a topological lensing hypothesis without needing to make any assumption on which spherical 3-manifold would correspond to the isometry which has been found. This is a practical advantage for observers interested in making simple analyses of observational data.

Moreover, as mentioned by Gausmann et al. (2001) in Eq. (15) of their paper, by embedding $S^{3}$ in $\mathbb{R}^{4}$, the isometry can be written as a combination of two simultaneous rotations in orthogonal 2-planes in $\mathbb{R}^{4}$. Hence, the rotations in both 2-planes also need to be whole number roots of the identity in $\mathbb{R}^{2}$, i.e. their angles need to be whole number fractions of $2 \pi$, since the covering space can only be covered once.

This implies a simple and rapid test for testing whether or not a numerically known isometry is a root of the identity.

To make this paper self-contained, the complete algebraic formulae needed for these calculations, given the celestial positions and redshifts of corresponding objects, are presented.

In Sect. 2.1, a reminder on the terminology regarding different types of pairs of topologically lensed objects is presented.

The condition that the isometry is a root of the identity is presented algebraically and discussed in Sect. 2.2. An abstract representation of the isometry is written in Sect. 2.2.1, while the four-dimensional matrix representations of pairs - embedded in Euclidean 4-space, $\mathbb{R}^{4}$ - and the resulting calculation of

\footnotetext{
${ }^{1}$ See http://en.wikipedia.org/wiki/Holonomy for a formal definition of holonomy group.
}

the isometry given the objects' sky positions and redshifts, is presented in Sect. 2.2.2. The condition that the isometry is a root of the identity is then presented in a form easy to calculate in Sect. 2.2.3.

In Sect. 2.3, the condition that the two rotation angles of the isometry are whole number roots of $2 \pi$ is presented. In Sect. 2.3.1, the ideal case in which the isometry happens to be expressed in a convenient basis is presented. In Sect. 2.3.2, the more realistic case of an isometry expressed in an arbitrary orthonormal basis is presented as an eigenplane problem (in analogy with eigenvectors): an Earth or Solar System or Galaxy based coordinate system is unlikely to be aligned with the eigenplanes of the isometry of comoving space - in the case that the comoving space we live in is a multiply connected, spherical 3-manifold.

A method of finding the eigenplanes is presented in Sect. 2.3.3, and a numerical implementation of this is shown in Sect. 2.4.1.

A summary is presented in Sect. 3.

For review papers on cosmic topology, see, e.g. Lachièze-Rey \& Luminet (1995); Luminet (1998); Starkman (1998); Luminet \& Roukema (1999); workshop proceedings are in Starkman (1998) and following articles, and Blanlœil \& Roukema (2000). For comparison and classification of different observational strategies, see e.g. Uzan et al. (1999a); Luminet \& Roukema (1999); Roukema (2002); Rebouças \& Gomero (2004).

\section{Definitions and calculation of the isometry}

\subsection{Cosmic crystallography: Local isometries (type I pairs) vs. generator pairs (type II)}

The isometry between two images of a single region of physical space yields two types of pairs of objects which can reveal the isometry, and uncorrelated pairs:

(I) local or type I pairs which should occur multiple times independently of curvature;

(II) generator or type II pairs, which only occur multiple times for a 3-manifold in which there are Clifford translations, i.e. in the flat and spherical cases;

(III) uncorrelated pairs, which can be called "type III" pairs.

These are schematically illustrated in Fig. 1.

Correspondingly, principles of detecting these pairs have been developed:

(I) The type I pairs can be collected, more generally, as local $n$-tuplets (Roukema 1996), or the two-point autocorrelation function of the two-point auto-correlation function, known as "collecting correlated pairs" (CCP) Uzan et al. (1999a,b) can be used. Gomero et al. (2002) noted these pairs as small deformations of the pair histogram, used for detecting type II pairs.

(II) In a pair separation histogram (PSH), type II pairs should, ideally, show up as sharp spikes - this is "cosmic crystallography" (Lachièze-Rey \& Luminet 1995; Lehoucq et al. 1996; Gomero et al. 2002). 

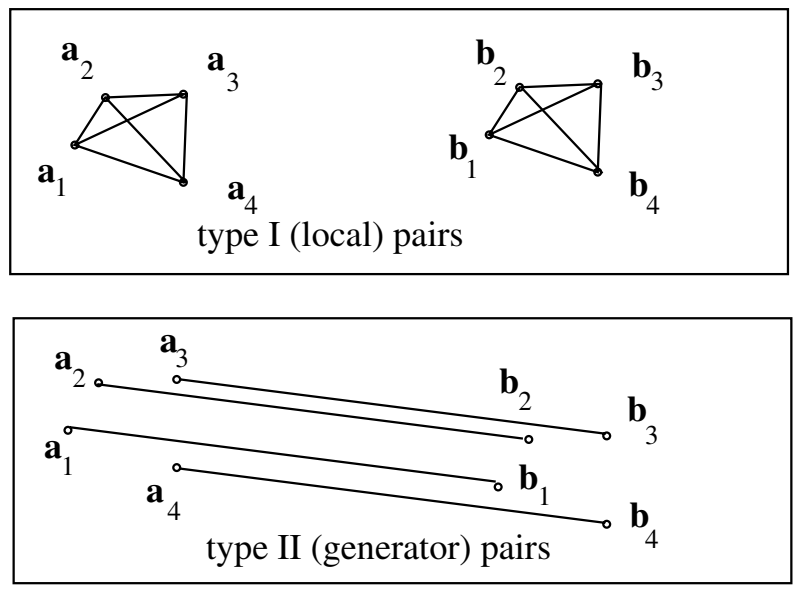

Fig. 1. Upper plot: example of $N=2$ images of a fundamental domain, where object $a_{i}$ is mapped to object $b_{i}$, i.e. $g\left(a_{i}\right)=b_{i}$. Each "local" (comoving spatial) geodesic between "local" objects should occur $N=2$ times, i.e. twice in this example. This frequency of occurrence is higher than for a Poisson distribution, in which any geodesic joining two objects should occur only once, not $N$ times. Lower plot: between the same two images of a fundamental domain, the geodesic connecting corresponding objects is a generator $g$ of the (physical) 3-manifold from the (apparent) covering space. If $g$ is a Clifford translation, then it occurs many times with (ideally) exactly the same length, again more frequently than that of a Poisson distribution.

As in most of observational cosmology, observed catalogues of objects are never as simple as could naïvely be hoped for making cosmological measurements. It could realistically be the case that a catalogue of objects contains a real topological signal, e.g. as in the right-hand plot of Fig. 15 of Gausmann et al. (2001), where $\left(\Omega_{\mathrm{m}}=0.35, \Omega_{\Lambda}=0.75\right)$, i.e. $\Omega_{\mathrm{tot}}=1.1$, detected either thanks to collecting together type I pairs or type II pairs, but that evolutionary and selection effects make the signal of only weak statistical significance.

While it is certainly possible to simply ignore such isometries which cannot be shown to be significant, it would good to be able to have some test which relates to their immediate geometrical properties and not to their membership of a statistical class.

This is the case presented in this paper: whether the suspected isometry is due to type I or type II pairs, if it is an isometry for the spherical case, then there does exist a simple test capable of falsifying the topological lensing hypothesis.

Let us use the notation shown in Fig. 1, where only one suspected realisation of the isometry $g$ is shown, mapping four points in one image of the fundamental domain to four points in another image:

$g:\left\{\boldsymbol{a}_{i}\right\}_{i=1,4} \rightarrow\left\{\boldsymbol{b}_{i}\right\}_{i=1,4}$

Although only three points are necessary to uniquely define an isometry $g$, four points are necessary if we wish to use the embedding in four-dimensional euclidean space as discussed below in Sect. 2.2.2. Given empirical uncertainties, it is probably useful to have the extra information provided by the fourth pair, which in the absence of observational uncertainties and numerical errors, would be redundant. The case of more than four points is a generalisation of this, including more redundant information.

\subsection{Root of the identity in $S^{3}$}

\subsubsection{Abstract representation}

We can then write the first condition in the case of $S^{3}$ which must be satisfied if $g$ really is an isometry of the covering space which generates a 3-manifold, and not just a coincidence:

$g^{n}(\boldsymbol{x}) \equiv g(g(\ldots g(\boldsymbol{x}) \ldots))=I$

where $I$ is the identity mapping, must be true for some whole number $n \in \mathbb{Z}$.

Moreover, the value of $n$ should not be so high that $g^{n}$ "goes past" one loop of $2 \pi$ around the hyper-sphere and evaluates to the identity after tiling the whole covering space twice or more. In other words, the tiling of $S^{3}$ by copies of the fundamental domain should only cover $S^{3}$ once.

To write this more formally, using a formalism which also enables the expression Eq. (2), in a way that is conceptually simple and easy to calculate, it is useful to embed $S^{3}$ in fourdimensional euclidean space, $\mathbb{R}^{4}$.

\subsubsection{4-D representations in $\mathbb{R}^{4}$ and calculation of the isometry}

By embedding $S^{3}$ in four-dimensional euclidean space, $\mathbb{R}^{4}$, the reader's intuition of $S^{2}$ embedded in three-dimensional euclidean space, $\mathbb{R}^{3}$ can be used.

The distance between two points (object locations) in comoving space can then be thought of as an arc-length in $\mathbb{R}^{4}$, along the 3-surface $S^{3}$ (e.g., Roukema 2001).

Let us write the $j$ th points of the $i=1,2$ members of the $j$ th pair of corresponding objects, i.e. where

$g\left(\boldsymbol{a}_{j}\right)=\boldsymbol{b}_{j}$

as

$\boldsymbol{a}_{j}=\left(\begin{array}{c}x_{1 j} \\ y_{1 j} \\ z_{1 j} \\ w_{1 j}\end{array}\right), \quad \boldsymbol{b}_{j}=\left(\begin{array}{c}x_{2 j} \\ y_{2 j} \\ z_{2 j} \\ w_{2 j}\end{array}\right)$,

evaluating these from, e.g. Eq. (33) of Roukema (2001), where the objects are located at celestial positions $\alpha_{i j}, \delta_{i j}$ and redshifts $s_{i j}$,

$\Omega_{\kappa} \equiv \Omega_{\mathrm{m}}+\Omega_{\Lambda}-1$

$R \equiv\left(c / H_{0}\right)\left(\Omega_{\kappa}\right)^{-0.5}$

$\chi_{i j}=\frac{c}{H_{0}} \int_{1 /\left(1+s_{i j}\right)}^{1} \frac{\mathrm{d} a}{a \sqrt{\Omega_{\mathrm{m}} / a-\Omega_{\kappa}+\Omega_{\Lambda} a^{2}}}$

$x_{i j}=R \sin \left(\chi_{i j} / R\right) \cos \delta_{i j} \cos \alpha_{i j}$

$y_{i j}=R \sin \left(\chi_{i j} / R\right) \cos \delta_{i j} \sin \alpha_{i j}$

$z_{i j}=R \sin \left(\chi_{i j} / R\right) \sin \delta_{i j}$

$w_{i j}=R \cos \left(\chi_{i j} / R\right)$. 
Since we are dealing with spherical spaces here, $\Omega_{\kappa}$ is positive.

In $\mathbb{R}^{4}$, the isometry $g$ between $\boldsymbol{a}_{j}$ and $\boldsymbol{b}_{j}$ is a rotation about the origin $(0,0,0,0)-S^{3}$ is the 3 -sphere (hypersphere) of radius $R$ centred on the origin, without loss of generality. This rotation can be written as a $4 \times 4$ matrix $M$ of unity determinant.

Since $M$ must map $\boldsymbol{a}_{j}$ to $\boldsymbol{b}_{j}$ for each $j$ (Eq. (3)), we have

$M A=B$

where $A$ and $B$ are the matrices

$A \equiv\left(\begin{array}{llll}a_{1} & a_{2} & a_{3} & a_{4}\end{array}\right), B \equiv\left(\begin{array}{llll}b_{1} & b_{2} & b_{3} & b_{4}\end{array}\right)$.

If the four-vectors in $\mathbb{R}^{4}$ of all four points $\boldsymbol{a}_{j}$ (equivalently, of $\boldsymbol{b}_{j}$ ) are linearly independent, then $A$ is invertible, and multiplication by $A^{-1}$ from the right-hand side of Eq. (6) gives

$M=B A^{-1}$,

which should be a matrix of nearly unity determinant, apart from the positional uncertainties which are considered "acceptable" for the calculation. Discussion of what precision is "acceptable" is postponed to Sect. 2.4.2 below.

Hence, $M$ can be calculated from the celestial positions and redshifts of the two pairs of four corresponding objects, together with the values of the curvature parameters for which the isometry was found.

$M$ is a matrix representation of the generator $g$.

However, even if all four points are distinct, their fourvectors in $\mathbb{R}^{4}$ are not necessarily linearly independent (e.g. 3 vectors could be aligned in the same 2-plane). Moreover, if the $\boldsymbol{a}_{j}$ are nearly, but not quite, aligned, then the system could be ill-conditioned, i.e. be highly sensitive to small errors. Any numerical application of this method should either ignore choices of 4-tuplets which are not linearly independent enough or at least flag them and warn the user.

\subsubsection{Euclidean 4-D representation of the root of the identity constraint}

The constraint on the generator $g \equiv M$ in Eq. (2) can now be rewritten

$M^{n}=I$.

Clearly, naïvely testing this numerically to arbitrarily large $n$ would not be a practical way to test this. This is because in the case of incorrect hypotheses, a computer making numerically exact (rather than approximate) calculations would require a (countably) infinite number of calculations in order to check that no $n$ is large enough to yield $M^{n}=I$. Real computers make approximations and are subject to numerical errors - as $n$ gets bigger, these errors would increase without limit and make the calculation meaningless at some large value of $n$, unless the algorithm recalculated each successive estimate of $M^{n}$ to higher and higher precision, at the expense of increasing the computing time per calculation as $n$ gets higher, ensuring no possibility of a convergent series for the total computing time requiring.

\subsection{Two rotation angles as roots of the 2-D identity}

\subsubsection{Constraint represented in a well-chosen basis}

However, as described using a four-dimensional matrix representation in Eq. (15) of Gausmann et al. (2001), there exists an orthonormal basis in which $M$ can be expressed in the form

$M=\left[\begin{array}{cccc}\cos \theta & -\sin \theta & 0 & 0 \\ \sin \theta & \cos \theta & 0 & 0 \\ 0 & 0 & \cos \phi & -\sin \phi \\ 0 & 0 & \sin \phi & \cos \phi\end{array}\right]$

where the rotation angles $\theta$ and $\phi$ clearly have to satisfy

$\frac{2 \pi}{\theta}, \frac{2 \pi}{\phi} \in \mathbb{Z}$.

Writing the least common multiple as

$n \equiv \operatorname{LCM}\left\{\frac{2 \pi}{\theta}, \frac{2 \pi}{\phi}\right\}$,

we then have the smallest value $n$ such that $M^{n}=I$. If either $\frac{2 \pi}{\theta}$ or $\frac{2 \pi}{\phi}$ are not integers, then clearly $M$ is not a root of the identity.

\subsubsection{Eigenplanes}

However, the orthonormal basis in $\mathbb{R}^{4}$ corresponding to a given astronomical coordinate system is unlikely, in general, to be the basis in which $M$ already has this form.

The representation of $M$ in Eq. (10) has four orthonormal basis vectors. Let us write these as

$s, t, u, v$

so that

$M \boldsymbol{s}=\cos \theta \boldsymbol{s}+\sin \theta \boldsymbol{t}$

$M \boldsymbol{t}=-\sin \theta \boldsymbol{s}+\cos \theta \boldsymbol{t}$

$M \boldsymbol{u}=\cos \phi \boldsymbol{u}+\sin \phi \boldsymbol{v}$

$M v=-\sin \phi \boldsymbol{u}+\cos \phi \boldsymbol{v}$.

In analogy with eigenvectors and eigenvalues, we can call this an eigenplane problem, where:

$M\left[\begin{array}{ll}\boldsymbol{s} \boldsymbol{t}\end{array}\right]=\left[\begin{array}{ll}\boldsymbol{s} \boldsymbol{t}\end{array}\right] \Lambda_{\theta}, \quad M[\boldsymbol{u} \boldsymbol{v}]=[\boldsymbol{u} \boldsymbol{v}] \Lambda_{\phi}$,

and

$\Lambda_{\theta}=\left[\begin{array}{cc}\cos \theta & -\sin \theta \\ \sin \theta & \cos \theta\end{array}\right], \quad \Lambda_{\phi}=\left[\begin{array}{cc}\cos \phi & -\sin \phi \\ \sin \phi & \cos \phi\end{array}\right]$,

i.e. the 1-dimensional (scalar) eigenvalue $\lambda$ of the traditional problem is replaced by a 2-dimensional eigenrotation $\Lambda_{\theta}$ or $\Lambda_{\phi}$.

Just as there is freedom up to multiplication by a scalar for the eigenvectors of an eigenvector problem, there is freedom up to rotation by an arbitrary (non-zero) rotation to find the eigenplane in the eigenplane problem. If $[\boldsymbol{s} \boldsymbol{t}]$ is an orthonormal basis representing one eigenplane of $M$, with eigenrotation $\Lambda_{\theta}$, then $[s \boldsymbol{t}] A$, where $A$ is an arbitrary 2-dimensional rotation matrix, is also an orthonormal basis for the same eigenplane and same eigenrotation:

$M\left[\begin{array}{ll}\boldsymbol{s} & \boldsymbol{t}\end{array}\right] A=\left[\begin{array}{ll}\boldsymbol{s} & \boldsymbol{t}\end{array}\right] A \Lambda_{\theta}$. 
In the particular case of interest here, there should exist two orthogonal eigenplanes in order for the isometry to correspond to a holonomy transformation, i.e. for it to be of interest for generating a 3-manifold as a quotient space of the 3-sphere centred at the origin of $\mathbb{R}^{4}$.

\subsubsection{Calculating the eigenplanes}

How do we find the basis vectors, now grouped into two pairs, $(\boldsymbol{s}, \boldsymbol{t}),(\boldsymbol{u}, \boldsymbol{v})$ spanning these two 2-planes respectively?

Since eigenvalue problems are normally solved using an iterative algorithm, it seems natural to develop a practical algorithm for solving the eigenplane problem.

Suppose that we already have one vector in one of the two eigenplanes $P_{1}$ or $P_{2}$. Then, without loss of generality (wlog), we can write this

$s \in P_{1}$

and $s$ and $M s$ can be used to construct a second vector in the same 2-plane, yielding a pair of orthonormal vectors $(s, t)$ :

$t \equiv \frac{M s-(s \cdot M s) s}{\sqrt{1-(s \cdot M s)^{2}}}$,

where is the inner product on $\mathbb{R}^{4}$.

It is clear that $s$ and $t$ are orthogonal, since

$$
\begin{aligned}
\boldsymbol{s} \cdot \boldsymbol{t} & =\frac{\boldsymbol{s} \cdot M \boldsymbol{s}-\boldsymbol{s} \cdot(\boldsymbol{s} \cdot M s) s}{\sqrt{1-(s \cdot M s)^{2}}} \\
& =\frac{(\boldsymbol{s} \cdot M s)(1-s \cdot s)}{\sqrt{1-(s \cdot M s)^{2}}}
\end{aligned}
$$

and $1-\boldsymbol{s} \cdot \boldsymbol{s}=0$ since $\boldsymbol{s}$ is a unit vector.

Next, we need a vector which is not in $P_{1}$. Consider three (orthonormal) basis vectors, $\boldsymbol{e}_{1}, \boldsymbol{e}_{2}, \boldsymbol{e}_{3}$, in the known basis, i.e. the basis in which $\boldsymbol{a}_{j}$ and $\boldsymbol{b}_{j}$ are calculated. Since these are orthogonal to one another, at most two of them can be in $P_{1}$. Choose one of these outside of $P_{1}$, let us call it $\boldsymbol{e}_{1}$ wlog. Then,

$u \equiv e_{1}-\left(e_{1} \cdot s\right) s-\left(e_{1} \cdot t\right) t$

$v \equiv \frac{M u-(\boldsymbol{u} \cdot M \boldsymbol{u}) \boldsymbol{u}}{\sqrt{1-(\boldsymbol{u} \cdot M \boldsymbol{u})^{2}}}$

are an orthonormal pair spanning the second plane, $P_{2}$.

We then have $\theta$ and $\phi$ from

$\theta=\cos ^{-1}(\boldsymbol{s} \cdot M \boldsymbol{s})$

$\phi=\cos ^{-1}(\boldsymbol{u} \cdot M \boldsymbol{u})$.

Hence, finding a solution for $(\boldsymbol{s}, \boldsymbol{t}),(\boldsymbol{u}, \boldsymbol{v})$ reduces to finding at least one vector $s$ in one of the two planes $P_{1}, P_{2}$.

The intersection of $P_{1}$ (or $P_{2}$ ) with $S^{3}$ is a great circle, centred on the origin. This must intersect somewhere with the 2-sphere defined by

$S_{123}^{2} \equiv \operatorname{span}\left\{\boldsymbol{e}_{1}, \boldsymbol{e}_{2}, \boldsymbol{e}_{3}\right\} \cap S^{3}$.

Hence, it is sufficient to search through $S_{123}^{2}$ looking for at least one vector which lies in $P_{1}$ or $P_{2}$. In fact, it is sufficient to search through half of this region, since polarity is unimportant.
To write down the condition for a vector $s$ to be in one of the two eigenplanes, note that the three vectors

$\boldsymbol{s}, M s, M^{2} s$,

must all lie in the same eigenplane. This eigenplane intersects with $S^{3}$ in a great circle, i.e. rotating twice corresponds to a single rotation by twice the angle of the original rotation. We can write this condition as

$\cos ^{-1}\left(s \cdot M^{2} s\right)=2 \cos ^{-1}(s \cdot M s)$

for a rotation angle

$\theta \equiv \cos ^{-1}(s \cdot M s) \leq \pi / 2$

or

$2 \pi-\cos ^{-1}\left(s \cdot M^{2} s\right)=2 \cos ^{-1}(s \cdot M s)$,

for

$\pi / 2 \leq \theta \equiv \cos ^{-1}(s \cdot M s) \leq \pi$

Hence, an iterative search to sucessively preciser resolution through $S_{123}^{2}$ to find a vector $s$ satisfying either Eq. (25) or Eq. (27) to the desired numerical precision yields one basis vector $\boldsymbol{s}$ of the eigenplanes, and Eqs. (19), (21) yield the other three basis vectors.

Tests using the eigenplane package (Sect. 2.4.1) suggest that the number of steps $n$ to reach a precision in radians of $\Delta \theta$ is

$n=2-4 \log _{10} \Delta \theta$,

i.e. for $\Delta \theta \sim 10^{-4}-10^{-3}$, about 14-18 iterations are sufficient.

The rotation angles corresponding to the isometry are those in Eq. (22).

If these angles do not satisfy Eq. (11), then the isometry is not a root of the identity and the topological lensing hypothesis is false.

\subsection{Numerical aspects}

\subsubsection{Numerical implementation}

The package eigenplane, which is a free software (GNU GPL licence) implentation of an iteration algorithm to generate the two rotation angles $\theta, \phi$ (and as a side product, a choice of four orthonormal vectors $(\boldsymbol{s}, \boldsymbol{t}),(\boldsymbol{u}, \boldsymbol{v})$ defining the two eigenplanes), given an input isometry $M$, has been prepared and is available for download at http: //cosmo. torun.pl/GPLdownload/eigen/2 .

The package is self-contained, apart from requiring fortran and $\mathrm{C}$ compilers and the public domain linear algebra library blas. Installation (help in README and INSTALL files) is by the standard ./configure; make; make install sequence, including standard options such as a non-root user installation directory via --prefix=PREFIX. Following installation, help is available with the commands eigenplane --help and info eigenplane.

2 At the time of proofchecking this article, the current version of the package is eigenplane-0.2.3. Users are welcome to add features, correct bugs and distribute modified versions. 


\subsubsection{What are "acceptable" levels of errors in the positions?}

As was discussed, e.g. in Roukema (1996) and later papers, these consist, in principle, both of "measurement" errors - to what extent the observed values of angular positions $\alpha_{i j}, \delta_{i j}$ and redshift $s_{i j}{ }^{3}$ incorrectly represent the true position if peculiar velocity is ignored - and of "movement" error - to what extent the objects move relative to the comoving reference frame between the different epochs (redshifts) at which they are observed.

In practice, the precision on the angular positions is negligible compared to all other errors. The former are almost always more accurate than an arcsecond, which at a redshift of $s=2$ and $\left(\Omega_{\mathrm{m}}=0.35, \Omega_{\Lambda}=0.75\right)$ corresponds to a tangential error of better than $0.02 h^{-1} \mathrm{Mpc}$.

The measurement error in the redshifts, e.g. of quasars, can usually be obtained to $\Delta s<0.001$, but in big catalogues is more often $\Delta s<0.01$. Again at a redshift $s=2$ and $\left(\Omega_{\mathrm{m}}=\right.$ $\left.0.35, \Omega_{\Lambda}=0.75\right)$, these two errors lead to distance errors of about $1 h^{-1} \mathrm{Mpc}$ and $10 h^{-1} \mathrm{Mpc}$ respectively, at least 50 times larger than that induced by any angular error.

The movement error between observations at different redshifts depends on how different the redshifts are, and on hypotheses of structure formation within the general model of gravitational collapse from linear perturbations. The objects most likely to be observed are the brighter ones, which tend to lie in the heavier potential wells, which can be expected to move more slowly relative to the comoving reference frame.

If we estimate a maximum for the peculiar velocity as a mean of $400 \mathrm{~km} \mathrm{~s}^{-1}$ then between two substantially different redshifts, e.g. differing by $4 \mathrm{Gyr}$, the total displacement (relative to the comoving reference frame) is about $1.6 h^{-1} \mathrm{Mpc}$. Moreover, simulations suggest that some objects may "stream" from voids towards filaments and along filaments towards the deepest potential wells in the cosmic web of gravitationally bound structure - i.e. movement by up to a few megaparsecs over a big fraction of a Hubble time is realistic for objects forming some distance away from the biggest clusters of galaxies.

So likely errors are in the range $1-10 h^{-1} \mathrm{Mpc}$, both from redshift measurements and from possible movement relative to the comoving frame.

How much do these affect testing an isometry as a root of the identity?

Again, for $\left(\Omega_{\mathrm{m}}=0.35, \Omega_{\Lambda}=0.75\right)$, the curvature radius is $R \approx 10 h^{-1} \mathrm{Gpc}$, so the errors in three-dimensional position, i.e. within the 3-surface $S^{3}$, are at the level of about $10^{-4}$ to $10^{-3}$ curvature radii, i.e. about $10^{-4}$ to $10^{-3}$ radians. If these lead to a similar error in the estimates of the angles $\theta$ and $\phi$, and the magnitude of $\max (\theta, \phi)$ is a big fraction of the distance to the surface of last scattering, e.g. $\geq 5 h^{-1} \mathrm{Gpc} \sim 0.5$ radians, then the fractional (relative) errors in $\max \left(\frac{2 \pi}{\theta}, \frac{2 \pi}{\phi}\right)$ should be of the same order of magnitude, about $10^{-4}$ to $10^{-3}$. A reasonable lower bound could be placed on $\max (\theta, \phi)$, for a given $\left(\Omega_{\mathrm{m}}, \Omega_{\Lambda}\right)$ pair, in order for the isometry to be realistic

\footnotetext{
${ }^{3}$ The usual variable $z$ is used here for positions in $\mathbb{R}^{4}$, so we write $s$ (red-"shift") instead.
}

(e.g. given cosmic topology constraints from the cosmic microwave background), removing isometries where $\max (\theta, \phi)$ is too small. So, for holonomy groups of order up to $\sim 10^{1}$, the probability of false (chance) isometries yielding integer solutions is about $10^{-3}$ to $10^{-2}$. For larger orders, the probability of false solutions necessarily becomes higher.

It remains possible that the smaller angle, $\min (\phi, \theta)$, could be quite small and would have a larger relative error (e.g. $\left.10^{-2}-10^{-1}\right)$, so in many cases would constitute a weaker test of candidate isometries.

If the values $\left(\Omega_{\mathrm{m}}, \Omega_{\Lambda}\right)$ are decreased to approach $\Omega_{\mathrm{m}}+$ $\Omega_{\Lambda}=1$, then the curvature radius $R$ increases rapidly but the distance to the surface of last scattering only changes slightly, so $\max (\theta, \phi)$ decreases. This causes $\max \left(\frac{2 \pi}{\theta}, \frac{2 \pi}{\phi}\right)$ to increase rapidly, so even while the relative error will not increase, the absolute error will increase to above \pm 1 , in which case integer solutions will necessarily be found for chance isometries.

However, as pointed out by Mota et al. (2005), as the curvature radius becomes larger and larger, observationally detectable isometries in spherical (and hyperbolic) 3-manifolds become closer and closer to those for the flat case, in which case methods used in the flat case are likely to become preferable.

\section{Discussion and conclusion}

Of course, checking that an isometry is a root of the identity is only a necessary condition for the topological lensing hypothesis to be correct, it is not sufficient. Moreover, even if it genuinely is a case of topological lensing, the isometry might, in principle, not be an isometry between adjacent copies of the fundamental domain - or in other words, the group it generates as the $n$-th root of the identity might only be a sub-group of the full holonomy group (Weeks et al. 2003).

Note that there is no point checking the Poincaré dodecahedral hypothesis of Luminet et al. (2003) and Roukema et al. (2004) by this method, since the isometries there are already known to be roots of the identity; the assumption of a particular class of 3-manifolds was an input assumption.

The situations where this test can be useful are those where a candidate isometry is obtained for the spherical case without having assumed any particular 3-manifold, but only having assumed that curvature is positive.

How can these candidate isometries be obtained? In other words, what are the known systematic methods for exploring a catalogue of objects extending to high redshifts in order to find a suitable pair of 4-tuplets of corresponding objects with a corresponding $g$ which should be tested?

In principle, the method of finding local $n$-tuplets of Roukema (1996) provides the answer: compare all "local" (less than a few hundred Mpc) "configurations" of objects against all other "local clusters", each time checking whether the mapping $f$ between the two 4-tuplets is an isometry $g$ or not. In practice, optimising the algorithm for application to large, recent catalogues is a non-trivial task, since the number of $n$-tuplets climbs rapidly as the number of objects increases: the naïve approach with a large catalogue quickly becomes impractical even on the latest supercomputers, though some 
suggestions for shortcuts to the algorithm are made in Roukema (1996).

A related approach might be to use the "collecting correlated pairs" method (CCP) (Uzan et al. 1999a,b; Gomero et al. 2002), to first obtain a list of 2-tuplets (pairs) "most likely to be matched type I pairs", then try to combine these into 4-tuplets, test whether or not the mappings $f$ are isometries $g$, and finally test whether any "best" isometry $g$ is a root of the identity, using the algorithm presented here. The definition of the "most likely to be matched type I pairs" might first be some way of choosing the highest signal-to-noise ratios as a function of the curvature parameters $\left(\Omega_{\mathrm{m}}, \Omega_{\Lambda}\right)$ (as is recommended in Uzan et al. 1999a,b), and then for each choice of $\left(\Omega_{\mathrm{m}}, \Omega_{\Lambda}\right)$, choose the bins in the pair separation histogram with the highest numbers of pairs as the "most likely to be matched type I pairs".

Whereas Uzan et al. (1999a,b) hope for a strong signal, use of the algorithm presented here could potentially enable detection of a candidate 3-manifold even if the signal is weak.

Type II pairs - generator pairs - detected as high spikes in a pair separation histogram (PSH) (Lachièze-Rey \& Luminet 1995; Lehoucq et al. 1996; Gomero et al. 2002), could also be used to generate pairs of 4-tuplets and isometries $g$.

Another caveat is that this test necessarily depends on the choice of curvature parameters $\left(\Omega_{\mathrm{m}}, \Omega_{\Lambda}\right)$.

If an isometry $g$ is known numerically based on, e.g. a pair of 4-tuplets of observed objects for a given choice of $\left(\Omega_{\mathrm{m}}, \Omega_{\Lambda}\right)$, then it is fairly likely that the same pair of 4-tuplets will be approximately isometric for nearby values of $\left(\Omega_{\mathrm{m}}, \Omega_{\Lambda}\right)$, especially if the 4-tuplets are local 4-tuplets (composed of type I pairs rather than type II pairs). So, the range of $\left(\Omega_{\mathrm{m}}, \Omega_{\Lambda}\right)$ for which the mapping between a given pair of 4-tuplets remains an isometry needs to be tested, unless external constraints on $\left(\Omega_{\mathrm{m}}, \Omega_{\Lambda}\right)$ are considered acceptable. This (unfortunately) increases the chance that a false (chance) isometry will yield a valid root of the identity.

Acknowledgements. The author thanks an anonymous referee for many very constructive comments.

\section{References}

Blanlœil, V., \& Roukema, B. F. 2000, Cosmological Topology in Paris 1998, Paris [arXiv:astro-ph/0010170]
Chiang, L., Naselsky, P. D., Verkhodanov, O. V., \& Way, M. J. 2003, ApJ, 590, L65

Choudhury, T. R., \& Padmanabhan, T. 2003 [arXiv: astro-ph/0311622]

Copi, C. J., Huterer, D., \& Starkman, G. D. 2004, Phys. Rev. D, 70, 043515

Cornish, N. J., Spergel, D. N., Starkman, G. D., \& Komatsu, E. 2004, Phys. Rev. Lett., 92, 201302

Gausmann, E., Lehoucq, R., Luminet, J.-P., Uzan, J.-P., \& Weeks, J. 2001, Classical and Quantum Gravity, 18, 5155

Gomero, G. I., Teixeira, A. F. F., Rebouças, M. J., \& Bernui, A. 2002, Int. J. Mod. Phys. D, 11, 869

Lachièze-Rey, M., \& Luminet, J. 1995, Phys. Rep., 254, 135

Lehoucq, R., Lachièze-Rey, M., \& Luminet, J.-P. 1996, A\&A, 313, 339

Luminet, J., \& Roukema, B. F. 1999, in NATO ASIC Proc. 541, Theoretical and Observational Cosmology, 117

Luminet, J., Weeks, J. R., Riazuelo, A., Lehoucq, R., \& Uzan, J. 2003 , Nature, 425, 593

Luminet, J.-P. 1998, Acta Cosmol., XXIV-1, 105 [arXiv:gr-qc/9804006]

Mota, B., Reboucas, M. J., \& Tavakol, R. 2005 [arXiv: astro-ph/0503683]

Myers, A. D., Shanks, T., Outram, P. J., Frith, W. J., \& Wolfendale, A. W. 2004, MNRAS, 347, L67

Perlmutter, S., Aldering, G., Goldhaber, G., et al. 1999, ApJ, 517, 565

Rebouças, M. J., \& Gomero, G. I. 2004, Braz. J. Phys., 34, 1358

Roukema, B. F. 1996, MNRAS, 283, 1147

Roukema, B. F. 2001, MNRAS, 325, 138

Roukema, B. F. 2002, in Marcel Grossmann IX Conference on General Relativity, ed. V. G. Gurzadyan, R. T. Jantzen, \& R. Ruffini, (Singapore: World Scientific), 1937

[arXiv: astro-ph/0010189]

Roukema, B. F., Lew, B., Cechowska, M., Marecki, A., \& Bajtlik, S. 2004, A\&A, 423, 821

Schwarzschild, K. 1900, Vier. d. Astr. Gess, 35, 337

Schwarzschild, K. 1998, Classical and Quantum Gravity, 15, 2539

Spergel, D. N., Verde, L., Peiris, H. V., et al. 2003, ApJS, 148, 175

Starkman, G. D. 1998, Classical and Quantum Gravity, 15, 2529

Tegmark, M., de Oliveira-Costa, A., \& Hamilton, A. 2003, Phys. Rev. D, 68, 123523

Uzan, J.-P., Lehoucq, R., \& Luminet, J.-P. 1999a, in Proc. of the XIXth Texas meeting, Paris 14-18 December 1998, ed. E. Aubourg, T. Montmerle, J. Paul, \& P. Peter, article No. 04/25 [arXiv:gr-qc/0005128]

Uzan, J.-P., Lehoucq, R., \& Luminet, J.-P. 1999b, A\&A, 351, 766

Weeks, J., Lehoucq, R., \& Uzan, J. 2003, Classical and Quantum Gravity, 20, 1529 